\title{
Factores de riesgo y protección del suicidio en adultos mayores
}

\section{Risk and protection factors of suicide in older adults}

\author{
Laura Angélica Montes de Oca Valdez y David Alberto Rodríguez Medina ${ }^{1}$ \\ Citación: Montes de Oca V., L.A. y Rodríguez M., D.A. (2019). Factores de riesgo y protección \\ del suicidio en adultos mayores. Psicología y Salud, 29(2), 187-194.
}

\section{RESUMEN}

\begin{abstract}
La escasa publicación de trabajos acerca del suicidio en adultos mayores mexicanos genera una considerable falla en su prevención, misma que se refleja en las altas cifras que el fenómeno muestra. En el presente estudio se realizó una revisión a partir de una perspectiva biopsicosocial, utilizando para ello estudios hispanoamericanos reportados respecto a la detección de factores de riesgo y de protección. Tras una búsqueda en bases de datos especializadas, se seleccionaron nueve artículos publicados entre los años 2000 y 2018. El análisis de los citados artículos permitió elaborar una tabla de 49 variables, divididas en 28 factores de riesgo y 21 factores protectores, divididos a su vez en factores biológicos, psicológicos y sociales. Los resultados pueden considerarse una base para elaborar futuros estudios epidemiológicos y empíricos, a fin de proponer un modelo de prevención del suicidio en el adulto mayor.
\end{abstract}

Palabras clave: Conducta suicida; Vejez; Factores de riesgo-protección; Prevención.

\begin{abstract}
The scarcity of research publications on suicide in Mexican elderly people has resulted in prevention failure reflected in the high figures shown by this phenomenon. The present study involved a systematic review of the research literature within a bio-psycho-social perspective in published studies with Hispanic-American participants, regarding risk and protection factors. A search in specialized databases yielded nine articles published between 2000 and 2018. The data analysis, summarized in a table revealed 46 variables, including 28 risk factors and 21 protective factors; these were in turn subdivided into biological, psychological, and social factors. These results should be considered as basis for designing future epidemiological and empirical studies, and developing suicide prevention models for the elderly.
\end{abstract}

Key words: Suicidal behavior; Elderly; Risk-protection factors; Prevention.

\section{INTRODUCCIÓN}

$\mathrm{L}$ a Organización Mundial de la Salud (OMS) (2014) definió el suicidio como "todo acto por el que un individuo se causa a sí mismo una lesión, o un daño, con un grado variable de la intención de morir, cualquiera sea el grado de la intención letal o de conocimiento del verdadero móvil". Aproximadamente 800 mil personas se quitan la vida anualmente en todo el mundo, lo que convierte el suicidio en un grave problema de salud pública. En el año 2015 se ubicó como la segunda causa de defunción en el grupo de 15 a 29 años (OMS, 2014), y en México cada año se registran mil 500 suicidios, aunque se ha observado un aumento en esa tasa; en efecto, en el año 2000 era de 3.7 casos por cada 100 mil personas, pero en 2014 el índice aumentó a 4.9.

\footnotetext{
${ }^{1}$ Facultad de Psicología, Universidad Nacional Autónoma de México. Para correspondencia: Sector 38 Mz 123 Lt 15, Casa 3, 55763 Los Héroes

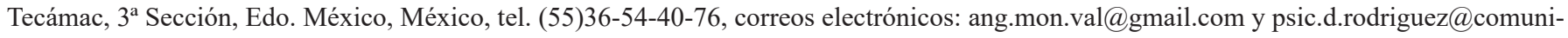
dad.unam.mx. Artículo recibido el 22 de mayo y aceptado el 11 de septiembre de 2018.
} 
El grupo poblacional con la mayor tasa de suicidio en México son los adultos de 20 a 24 años, con 852 casos registrados en 2013, en su mayoría mujeres, según estadísticas publicadas por el Instituto Nacional de Estadística y Geografía (INEGI) (2014); le sigue el grupo de edad de 15 a 19 años, con 760 suicidios, y en tercer lugar el grupo de jóvenes de 25 a 29 años, con 733 casos registrados (Cruz, 2017). Estas cifras centran los estudios sobre el suicidio únicamente en adultos jóvenes, pero dan escasa importancia a la conducta suicida en los adultos mayores, que también presentan el fenómeno, con un registro de alrededor de 300 fallecimientos al año en el grupo de personas de más de 60 años (INEGI, 2011).

La población mexicana muestra un creciente envejecimiento demográfico; según el INEGI (2014), el número de adultos mayores se duplicó en las últimas décadas, pasando de 5 millones a $11.7 \mathrm{mi}-$ llones entre 1990 y 2014. De acuerdo con las proyecciones de población, para el año 2025 habrá 17.2 millones, y 32.4 millones en 2050; sin embargo, de las 656 mil defunciones registradas en las estadísticas de mortalidad del INEGI en 2015, $65 \%$ correspondió a personas de 60 años y más (INEGI, 2017).

Gracias a los programas gubernamentales instrumentados para la atención, recuperación y preservación de la salud, la longevidad ha aumentado (OMS, 2012), aunque no se han puesto en práctica medidas específicas que puedan prever la calidad y la forma en que se experimentará esa etapa de la vida. El INEGI (2014) señala que las principales causas de estas muertes son las enfermedades crónicas, pero las personas mayores, además de un evidente deterioro físico, llegan a sufrir aislamiento, soledad y angustia, que en la mayoría de las ocasiones desembocan en cuadros depresivos que pueden conducirlas al suicidio.

De acuerdo al Instituto Mexicano del Seguro Social (IMSS, 2014), la principal causa del intento suicida en los adultos mayores es la depresión, desencadenada por la pérdida de vitalidad, el cambio en el ritmo de vida, la disminución de las funciones y capacidades (sumada al desempleo o la jubilación), la pérdida de figuras significativas (amigos, pareja, independencia de los hijos, distanciamiento con la familia), la aparición de enfermedades crónico-degenerativas e incluso el empeoramiento de la situación económica. Además, la citada institución señala que la falta de asistencia agrava el problema, pues no existe una atención integral adecuada para los adultos mayores. Aunado a ello, se encuentra la discriminación que sufre esta población -cuya percepción es tan importante como su misma experiencia- y sus efectos tanto en quienes la ejercen como en los que la padecen (Montes de Oca, 2013).

Debido a este panorama, la presente investigación, hecha a partir de una perspectiva biopsicosocial, exploró las variables que intervienen en la conducta suicida de los adultos mayores con el objetivo de identificar los factores de riesgo y protección reportados en artículos publicados en el periodo 2000-2018 en países hispanoamericanos.

\section{MÉTODO}

\section{Muestra}

La muestra utilizada consistió en artículos publicados en países hispanoamericanos entre los años 2000 y 2018 sobre la conducta suicida de los adultos mayores.

\section{Procedimiento}

Como unidades de análisis, la revisión se hizo en las bases de datos Dialnet, Redalyc y Scielo, utilizándose como núcleos temáticos los términos "suicidio" y "adultos mayores" (palabras clave). Como resultado, se recuperaron nueve artículos: uno de Dialnet, dos de Redalyc y seis de Scielo, mismos que se eligieron para configurar la citada muestra de artículos, los cuales debían cumplir el criterio de tener su origen en países hispanoamericanos.

\section{RESULTADOS}

La revisión de los nueve artículos permitió la elaboración de una tabla de resumen (Tabla 1), en la que se muestra su descripción general; dicha tabla contuvo las siguientes secciones: autor o autores, 
año y lugar de publicación, diseño de investigación, tipo y descripción de la muestra, instrumentos utilizados, medidas empleadas y resultados.

Dicha tabla muestra que los países de origen fueron Colombia, Cuba, España, México y
Uruguay; fue Cuba el país con más publicaciones, con un total de cuatro artículos, seguido por Colombia, con dos, y finalmente España, México y Uruguay, con uno.

Tabla 1. Resumen de los artículos seleccionados de 2000 a 2018.

\begin{tabular}{|c|c|c|c|c|c|c|c|}
\hline Autor(es) & Año & Lugar & Diseño & Muestra & Instrumento(s) & Medida(s) & Resultados \\
\hline Guibert y Sánchez & 2001 & Cuba & $\begin{array}{l}\text { Mixto. } \\
\text { Descriptivo- } \\
\text { transversal }\end{array}$ & $\begin{array}{l}26 \text { ancianos sin } \\
\text { deterioro cognitivo re- } \\
\text { sidentes del municipio } \\
10 \text { de Octubre de la } \\
\text { ciudad de La Habana, } \\
\text { con intento de suicidio } \\
\text { en los últimos tres } \\
\text { años. }\end{array}$ & $\begin{array}{l}\text { Entrevista semies- } \\
\text { tructurada de intento } \\
\text { suicida construida por } \\
\text { los autores y con vali- } \\
\text { dación de expertos. }\end{array}$ & $\begin{array}{l}\text { Características } \\
\text { sociodemográficas, } \\
\text { factores de riesgo, } \\
\text { razones del intento } \\
\text { suicida, factores } \\
\text { protectores, sistema } \\
\text { de apoyo social, tipo } \\
\text { y calidad de vida } \\
\text { percibida. }\end{array}$ & $\begin{array}{l}\text { Media edad, } 69.2 \text {, } \\
53.8 \% \text { mujeres, } 46.1 \% \\
\text { hombres, } 69.2 \% \text { con } \\
\text { escolaridad primaria, } \\
61.5 \% \text { jubilados, } 57.7 \% \\
\text { con ingresos económi- } \\
\text { cos medios. Factores de } \\
\text { riesgo más frecuentes: } \\
\text { sentimiento de desespe- } \\
\text { ranza (76.9\%), síndrome } \\
\text { depresivo ( } 76.9 \% \text { y } \\
\text { antecedente patológico } \\
\text { personal suicida (42.3\%). } \\
\text { Razón más prevalente del } \\
\text { intento suicida: conflictos } \\
\text { familiares (42.3\%). } \\
\text { Factores protectores más } \\
\text { frecuentes: bienestar } \\
\text { físico (76.9\%), red de } \\
\text { apoyo social instrumental } \\
\text { (53.8\%). }\end{array}$ \\
\hline $\begin{array}{l}\text { Gutiérrez, Gil, } \\
\text { Jiménez y Lugo }\end{array}$ & 2001 & Cuba & $\begin{array}{l}\text { Mixto. Des- } \\
\text { criptivo- } \\
\text { retrospectivo }\end{array}$ & $\begin{array}{l}\text { Defunciones por sui- } \\
\text { cidio en Manicaragua, } \\
\text { Villa Clara } \\
\text { entre } 1992 \text { y } 1999 .\end{array}$ & $\begin{array}{l}\text { Formulario de infor- } \\
\text { mación: medicina } \\
\text { legal, salud mental } \\
\text { y familiar. }\end{array}$ & $\begin{array}{l}\text { Características so- } \\
\text { ciodemográficas } \\
\text { y culturales. }\end{array}$ & $\begin{array}{l}77.5 \% \text { hombres, } 22.5 \% \\
\text { mujeres, } 37.5 \% \text {, media } \\
\text { de } 60-69 \text { años, } 35 \% \text { pen- } \\
\text { sionados, } 52.5 \% \text { solteros, } \\
55 \% \text { ambiente urbano, } \\
30 \% \text { en verano, } 75 \% \text { por } \\
\text { ahorcamiento, } 65 \% \text { en } \\
\text { estado depresivo. }\end{array}$ \\
\hline Casado & 2002 & España & $\begin{array}{l}\text { Mixto. } \\
\text { Revisión }\end{array}$ & $\begin{array}{l}\text { Defunciones por } \\
\text { suicidio en adultos } \\
\text { mayores de } 65 \text { años } \\
\text { en la provincia de } \\
\text { Badajoz. }\end{array}$ & $\begin{array}{l}\text { Estadísticas de } \\
\text { autopsias realizadas } \\
\text { por muertes violentas } \\
\text { en los diferentes } \\
\text { Partidos Judiciales } \\
\text { de la provincia de } \\
\text { Badajoz durante un } \\
\text { período de cinco años } \\
(1990-1995) \text {. }\end{array}$ & $\begin{array}{l}\text { Información del } \\
\text { médico forense, } \\
\text { familiares del adulto } \\
\text { mayor, informes e } \\
\text { historiales clínicos } \\
\text { en los archivos } \\
\text { de los diferentes } \\
\text { centros de salud y } \\
\text { hospitales. }\end{array}$ & $\begin{array}{l}37.93 \% \text { suicidios con- } \\
\text { sumados (de } 290,110 \\
\text { mayores de } 65 \text { años), } \\
\text { ligero aumento anual, } \\
83.6 \% \text { hombres, } 16.4 \% \\
\text { mujeres, } 49 \% \text { casados; } \\
\text { incidencia en días de la } \\
\text { semana, inferior en sába- } \\
\text { dos y domingos ( } 8.5 \% \text { ), } \\
\text { tasa de actos suicidas más } \\
\text { frecuentes en abril, mayo } \\
\text { y junio ( } 33.6 \% \text { ), } 81.8 \% \\
\text { en el propio domicilio, } \\
36.6 \% \text { con patologías psi- } \\
\text { quiátricas conocidas que } \\
\text { aparecieron como única } \\
\text { causa determinante. }\end{array}$ \\
\hline
\end{tabular}




\begin{tabular}{|c|c|c|c|c|c|c|c|}
\hline Sánchez y Gómez & 2002 & México & $\begin{array}{l}\text { Caso clínico. } \\
\text { Mixto. }\end{array}$ & $\begin{array}{l}\text { Hombre de } 54 \text { años, } \\
\text { intentos suicidas } \\
\text { recurrentes, cuadro } \\
\text { depresivo mayor y } \\
\text { diagnóstico de enfer- } \\
\text { medad de Huntington. }\end{array}$ & $\begin{array}{l}\text { Diagnósticos psiquiá- } \\
\text { tricos diferenciales, } \\
\text { exámenes de labora- } \\
\text { torio y neurológicos. }\end{array}$ & $\begin{array}{l}\text { Desempeño motor } \\
\text { y cognitivo. Salud } \\
\text { orgánica. }\end{array}$ & $\begin{array}{l}\text { La enfermedad causó } \\
\text { depresión y ansiedad, } \\
\text { pánico con predominio } \\
\text { nocturno, deterioro } \\
\text { paulatino en todas las } \\
\text { esferas (física, mental, } \\
\text { laboral, familiar, social } \\
\text { y ocio. Atrofia global del } \\
\text { cerebro con una pérdida } \\
\text { de } 24-30 \% \text { del peso del } \\
\text { encéfalo. Depresión } \\
\text { mayor (con intentos de } \\
\text { suicidio) desencadenada } \\
\text { por evento estresante (fue } \\
\text { atropellado) y malestar } \\
\text { físico. }\end{array}$ \\
\hline Portal y Montano & 2009 & Cuba & $\begin{array}{l}\text { Mixto. } \\
\text { Descriptivo } \\
\text { transversal }\end{array}$ & $\begin{array}{l}\text { Adultos mayores } \\
\text { fallecidos entre 2001- } \\
2005 \text { en la provincia } \\
\text { de Pinar del Río. }\end{array}$ & $\begin{array}{l}\text { Sistema de informa- } \\
\text { ción estadístico de } \\
\text { mortalidad, certifi- } \\
\text { cados médicos de } \\
\text { defunción, revisión } \\
\text { bibliográfica, consul- } \\
\text { ta a expertos. }\end{array}$ & $\begin{array}{l}\text { Edad, sexo, método } \\
\text { empleado para el } \\
\text { suicidio, hora de } \\
\text { ocurrencia, estrato } \\
\text { de la zona de resi- } \\
\text { dencia, tasas de mor- } \\
\text { talidad específica. }\end{array}$ & $\begin{array}{l}\text { Suicidio en descenso } \\
(63.5 \text { a } 43.3 \%) \text {, tasas } \\
\text { aumentaron con edad (60- } \\
69 \text { años } 23.7 \% ; 70-79 \text {, } \\
61.4 \% ; 80 \text { y más, } 73.9 \%) \text {. } \\
\text { El ahorcamiento fue el } \\
\text { método más empleado } \\
(31.8 \%) \text {, siendo más } \\
\text { frecuente en la primera } \\
\text { mitad del día (25.3\%), } \\
\text { mayor riesgo en zonas } \\
\text { rurales (60.6\%), mayor } \\
\text { incidencia en hombres } \\
(69 \%) \text { que en mujeres } \\
(15.7 \%) .\end{array}$ \\
\hline $\begin{array}{l}\text { Ribot, Alfonso, } \\
\text { Ramos y González }\end{array}$ & 2012 & Cuba & $\begin{array}{l}\text { Cualitativo. } \\
\text { Revisión } \\
\text { bibliográfica }\end{array}$ & $\begin{array}{l}\text { Adultos mayores con } \\
\text { conducta suicida. }\end{array}$ & $\begin{array}{l}\text { Libros, revistas, } \\
\text { documentos, sitios } \\
\text { web. }\end{array}$ & $\begin{array}{l}\text { Epidemiología del } \\
\text { suicidio en ancianos, } \\
\text { conducta suicida y } \\
\text { sus variantes. }\end{array}$ & $\begin{array}{l}\text { Diversas definiciones y } \\
\text { variantes del suicidio. } \\
\text { Estadísticas anuales (1,1 } \\
\text { millones al año, mayoría } \\
\text { de adultos de más de } 60 \\
\text { años). Factores de mayor } \\
\text { riesgo: ser varón, mayor } \\
\text { de } 60 \text { años, historia de un } \\
\text { intento previo, anteceden- } \\
\text { tes familiares de suicidio } \\
\text { o trastornos del estado del } \\
\text { ánimo, pérdida reciente } \\
\text { de pareja con gran carga } \\
\text { afectiva, aislamiento } \\
\text { social, dificultades econó- } \\
\text { micas, desempleo, senti- } \\
\text { mientos de incomprensión } \\
\text { reales o imaginarios, } \\
\text { humillaciones sociales y } \\
\text { drogadicción. }\end{array}$ \\
\hline
\end{tabular}




\begin{tabular}{|c|c|c|c|c|c|c|c|}
\hline Velásquez & 2013 & Colombia & $\begin{array}{l}\text { Cualitativo. } \\
\text { Revisión } \\
\text { bibliográfica }\end{array}$ & Adultos mayores. & $\begin{array}{l}\text { Bases de datos: } \\
\text { Pubmed, Scielo, oms, } \\
\text { Departamento Admi- } \\
\text { nistrativo Nacional de } \\
\text { Estadística, Instituto } \\
\text { Nacional de Medicina } \\
\text { Legal y Ciencias } \\
\text { Forense, Estudios } \\
\text { Nacionales de Salud } \\
\text { Mental } 1993 \text { y } 2003 \\
\text { de Colombia. }\end{array}$ & $\begin{array}{l}\text { Palabras clave: } \\
\text { suicidio, intento de } \\
\text { suicidio y anciano. }\end{array}$ & $\begin{array}{l}\text { Personas mayores de } 65 \\
\text { años presentan } 1,5 \text { veces } \\
\text { más riesgo de cometer } \\
\text { suicidios que jóvenes. } \\
\text { Factores de riesgo: } \\
\text { consumo de sustancias } \\
\text { psicoactivas, ideación } \\
\text { suicida, trastorno de } \\
\text { pánico, trastorno depre- } \\
\text { sivo, pérdidas recientes } \\
\text { (personales, laborales, } \\
\text { de salud, económicas), } \\
\text { predisposición a manifes- } \\
\text { tar actitud disfuncional, } \\
\text { como desesperanza, } \\
\text { aislamiento social o } \\
\text { demencias. Los ancianos } \\
\text { usan métodos más letales } \\
\text { para el suicidio que los } \\
\text { jóvenes (ahorcamien- } \\
\text { to, disparo con arma } \\
\text { de fuego, ingestión de } \\
\text { tóxicos, intoxicación con } \\
\text { monóxido de carbono. }\end{array}$ \\
\hline $\begin{array}{l}\text { Fachola, Lucero, } \\
\text { Porto, Díaz y París }\end{array}$ & 2015 & Uruguay & $\begin{array}{l}\text { Cualitativo. } \\
\text { Interpreta- } \\
\text { tivo. }\end{array}$ & $\begin{array}{l}\text { Cuatro ancianos } \\
\text { mayores de } 65 \text { años, } \\
\text { institucionalizados } \\
\text { en el Hospital Centro } \\
\text { Geriátrico "Dr. Pi- } \\
\text { ñeyro del Campo, con } \\
\text { historias de intentos de } \\
\text { autoeliminación. }\end{array}$ & $\begin{array}{l}\text { Entrevistas semies- } \\
\text { tructuradas, revisión } \\
\text { bibliográfica, nor- } \\
\text { mativas y acciones } \\
\text { preventivas de las } \\
\text { autoridades de salud } \\
\text { pública. }\end{array}$ & $\begin{array}{l}\text { Historias clínicas, } \\
\text { relación entre } \\
\text { tentativa e ideación } \\
\text { suicida, enfermedad } \\
\text { mental y física y } \\
\text { factores sociales. }\end{array}$ & $\begin{array}{l}\text { Dos casos con anteceden- } \\
\text { tes de autoeliminación } \\
\text { recientes, uno con difi- } \\
\text { cultades para hablar del } \\
\text { intento suicida, vergüenza } \\
\text { asociada a la vejez. Ma- } \\
\text { yor ideación en mujeres, } \\
\text { (intentos ocurridos en el } \\
\text { hogar con consumo de } \\
\text { sustancias); en los hom- } \\
\text { bres, intentos públicos } \\
\text { (arrojarse de puente o } \\
\text { frente a un vehículo). } \\
\text { Detonantes: situación } \\
\text { económica relacionada } \\
\text { con baja escolaridad, } \\
\text { conflictos familiares, } \\
\text { alcoholismo, violen- } \\
\text { cia, aislamiento social, } \\
\text { soledad, autonegligencia, } \\
\text { depresión. }\end{array}$ \\
\hline $\begin{array}{l}\text { Andrade, Lozano, } \\
\text { Rodríguez y } \\
\text { Campos }\end{array}$ & 2016 & Colombia & $\begin{array}{l}\text { Cualitativo. } \\
\text { Revisión } \\
\text { bibliográfica }\end{array}$ & $\begin{array}{l}\text { Adultos mayores } \\
\text { colombianos como po- } \\
\text { blación en crecimiento } \\
\text { con altos índices de } \\
\text { suicidio. }\end{array}$ & $\begin{array}{l}\text { Modelo de estrés-diá- } \\
\text { tesis, interacción de } \\
\text { factores predisponen- } \\
\text { tes y desencadenantes } \\
\text { en los niveles bioló- } \\
\text { gico, psicológico y } \\
\text { social. }\end{array}$ & $\begin{array}{l}\text { Influencia de la } \\
\text { vulnerabilidad psico- } \\
\text { social en el suicidio } \\
\text { de adultos mayores. }\end{array}$ & $\begin{array}{l}\text { Se considera el suici- } \\
\text { dio como un fenómeno } \\
\text { multifactorial, y al adulto } \\
\text { mayor como altamente } \\
\text { vulnerable a conductas } \\
\text { suicidógenas debido al } \\
\text { deterioro físico, cognitivo } \\
\text { y emocional que trae con- } \\
\text { sigo el envejecer en un } \\
\text { entorno de exclusión afec- } \\
\text { tiva y social constante. } \\
\text { Muchos casos de suicidio } \\
\text { se deben al abandono, } \\
\text { maltrato y desprecio. }\end{array}$ \\
\hline
\end{tabular}


La sección de resultados de la Tabla 1 permitió la elaboración de la Tabla 2. En esta última se clasifican las variables que, detectadas por los autores, intervienen en el suicidio de los adultos mayores, las cuales se dividieron en factores de riesgo y factores de protección, según los componentes del modelo biopsicosocial (cf. Ramos, 2015). Dicha tabla hizo posible identificar y agrupar las variables de mayor interés en los estudios publicados en países hispanoamericanos.

Tabla 2. Variables extraídas de los artículos y clasificadas en factores de riesgo y factores de protección del suicidio en adultos mayores según el modelo biopiscosocial.

\begin{tabular}{|c|c|c|c|}
\hline Factores & Biológico & Psicológico & Social \\
\hline Protección & $\begin{array}{l}\text { Actividad física. } \\
\text { Atención personalizada. } \\
\text { Autocuidado. } \\
\text { Buena alimentación. } \\
\text { Compensación de capacidades. } \\
\text { Funcionalidad. } \\
\text { Higiene. } \\
\text { Potencialización de capacidades. } \\
\text { Salud física. }\end{array}$ & $\begin{array}{l}\text { Autoestima. } \\
\text { Bienestar emocional. } \\
\text { Flexibilidad y adaptación a los cambios. } \\
\text { Motivación. } \\
\text { Planificación (segundo proyecto de vida). } \\
\text { Soporte emocional. }\end{array}$ & $\begin{array}{l}\text { Actividades recreativas. } \\
\text { Ambientes protésicos (que se } \\
\text { adapten o sean congruentes con las } \\
\text { capacidades de la persona). } \\
\text { Apoyo familiar. } \\
\text { Apoyo informal o formal. } \\
\text { Interacción grupal. } \\
\text { Redes de apoyo. }\end{array}$ \\
\hline Riesgo & $\begin{array}{l}\text { Antecedentes familiares de con- } \\
\text { ducta suicida. } \\
\text { Autonegligencia. } \\
\text { Demencias. } \\
\text { Discapacidades. } \\
\text { Enfermedades crónico-degene- } \\
\text { rativas. } \\
\text { Enfermedades crónicas no tras- } \\
\text { misibles. } \\
\text { Hospitalizaciones recurrentes. } \\
\text { Incapacidades. }\end{array}$ & $\begin{array}{l}\text { Conflictos familiares. } \\
\text { Consumo de sustancias psicoactivas. } \\
\text { Edad avanzada (de } 65 \text { años en adelante). } \\
\text { Eventos traumáticos (accidentes). } \\
\text { Ocio. } \\
\text { Pérdidas. } \\
\text { Sentimientos de desesperanza o incom- } \\
\text { prensión. } \\
\text { Sexo masculino. } \\
\text { Trastornos del estado de ánimo (depre- } \\
\text { sión, ansiedad). }\end{array}$ & $\begin{array}{l}\text { Abandono. } \\
\text { Aislamiento. } \\
\text { Ambiente demasiado protector, } \\
\text { complejo o desafiante. } \\
\text { Baja escolaridad. } \\
\text { Bajos ingresos. } \\
\text { Desempleo. } \\
\text { Discriminación. } \\
\text { Jubilación. } \\
\text { Malos tratos: abandono, negligen- } \\
\text { cia, abuso, violencia. }\end{array}$ \\
\hline
\end{tabular}

Se observa que en el abordaje para la elaboración de los estudios se utilizaron con mayor frecuencia los diseños mixtos, esto es, una combinación de metodologías cualitativas y cuantitativas en un total de cinco artículos, en tanto que en cuatro se empleó uno cualitativo; de hecho, no se encontró ningún estudio que fuera únicamente cuantitativo.

Se obtuvieron asimismo las frecuencias de la tabla de variables que se muestran en la Tabla 3.

Tabla 3. Frecuencias de las variables extraídas de los artículos.

\begin{tabular}{|l|c|c|c|c|}
\hline Variables & Biológicas & Psicológicas & Sociales & Total \\
\hline Protección & 9 & 6 & 6 & 21 \\
\hline Riesgo & 8 & 9 & 11 & 28 \\
\hline Total & 17 & 15 & 17 & 49 \\
\hline
\end{tabular}

\section{DISCUSIÓN}

En los artículos hallados se observó un considerable interés en este campo de estudio por de- terminar los factores de riesgo de la conducta suicida en los adultos mayores, subestimando sin embargo los factores de protección. A pesar de que los citados estudios destacan un aumento significativo de la población adulta mayor y de los casos de suicidio en dicha población, falta en México más investigación epidemiológica con un enfoque biopsicosocial de los aspectos que asume la conducta suicida esas personas. No obstante, los artículos que se reportan en esta investigación permiten acercarse al fenómeno y ofrecen una oportunidad de explorar los factores descritos empíricamente, e incluso desarrollar estudios longitudinales. Dar un seguimiento integral a los adultos mayores permitirá evaluar el proceso de envejecimiento y su relación con las conductas suicidas de forma integral y no sólo descriptiva.

El fenómeno aún está en proceso de reconocerse como un problema de salud pública. De hecho, se evidencia un mayor porcentaje de suicidios e intentos suicidas en los hombres, al contrario de lo que ocurre entre la población adulta joven, al menos en este país. De cualquier manera, 
recuperar los factores de protección reportados en los artículos aquí detallados fomenta un tipo de investigación orientada a la prevención y a la elaboración de programas y estrategias con un enfoque biopsicosocial que promuevan y exploten al máximo dichos factores, lo que puede disminuir la conducta suicida en la población analizada partiendo de la comunidad, la familia o el propio individuo en riesgo.

Actualmente hay en el país programas gubernamentales enfocados a la población anciana, como el Instituto Nacional de las Personas Adultas Mayores (INAPAM), que busca garantizar que los derechos humanos de la citada población se respeten plenamente; sin embargo, sus diversas acciones ponen el acento en la asistencia social y en la detección, control y seguimiento de enfermedades comunes (INAPAM, 2018). El apoyo brindado por este tipo de programas no considera el aspecto psicológico preventivo como una pieza angular; únicamente intenta disminuir los riesgos biológicos y sociales, pero omite la promoción de los factores de protección. Esa podría ser una razón por la que los citados programas no tienen un efecto visible en la disminución de las cifras de suicidio en los adultos mayores. La propuesta, entonces, es que a partir de la investigación empírica puedan impulsarse los factores protectores, principalmente desde el abordaje biopsicosocial.
Si bien la vejez implica un deterioro del individuo, no significa que quienes se hallan en esta etapa deban representar un papel secundario en la atención sanitaria. Es fundamental que tengan una buena calidad de vida, para lo cual bastaría con mejorar la prevención en la mayoría de los casos, es decir, promover los factores de protección desde las etapas previas a la vejez, evitando así condiciones acumuladas que propicien el suicidio como única solución. La psicología puede brindar las herramientas necesarias para la implementación de estas acciones, y sobre todo fomentar el apoyo a partir de un acompañamiento psicológico integral.

En conclusión, la investigación publicada sobre la conducta suicida en el adulto mayor es escasa en el contexto hispanoamericano. Sin embargo, los estudios muestran con claridad los factores de riesgo y protección que se pueden estudiar mediante la investigación empírica y epidemiológica. Desde una perspectiva biopsicosocial, es posible identificar los factores afectivos de riesgo que pueden precipitar dicho fenómeno, así como los protectores que propicien una mejor calidad de vida. Se sugiere para ello utilizar una metodología mixta que permita valorar y documentar los referidos factores en la población mexicana para así desarrollar nuevos modelos de prevención.

\section{REFERENCIAS}

Andrade S., J., Lozano S., L. Rodríguez R., A. y Campos R., A. (2016). Vulnerabilidad biopsicosocial y suicidio en adultos mayores colombianos. Revista Cultura del Cuidado, 13(1), 70-89.

Casado B., M. (2002). Suicidios en mayores de 65 años en la provincia de Badajoz: análisis médico legal. Cuadernos De Medicina Forense, 28(abril), 25-31. doi: 10.4321/s1135-76062002000200003.

Cruz, F. (2017). “Alertan alza en suicidios; jóvenes, los más vulnerables”. Periódico Excélsior (México), 2 de abril. Recuperado de http:/www.excelsior.com.mx/comunidad/2017/04/02/1155441.

Fachola, M., Lucero, R., Porto, V., Díaz, E. y París, M. (2015). Tentativa e ideación de suicidio en adultos mayores en Uruguay. Ciência y Saúde Coletiva, 20(6), 1693-1702. doi: 10.1590/1413-81232015206.02252015.

Guibert R., W. y Sánchez C., L. (2001). Ancianos con intento suicida en el municipio 10 de Octubre. Revista Cubana de Higiene y Epidemiología, 39(2), 126-135.

Gutiérrez Z., G., Gil R., I., Jiménez A., V. y Lugo J., B. (2001). Suicidio en la tercera edad: un problema de salud comunitario. Revista Cubana de Higiene y Epidemiología, 39(2), 147-151.

Instituto Mexicano del Seguro Social (2014). Depresión en el adulto mayor. México: IMSS. Recuperado de http://www.imss.gob. $\mathrm{mx} / \mathrm{salud}$-en-linea/preguntas-de-salud/depresion-adultomayor.

Instituto Nacional de Estadística y Geografía (2011). Estadística de suicidios de los Estados Unidos Mexicanos 2011. México: INEGI. Recuperado de http://internet.contenidos.inegi.org.mx/contenidos/Productos/prod_serv/contenidos/espanol/bvinegi/productos/continuas/sociales/suicidio/2011/702825047436.pdf. 
Instituto Nacional de Estadística y Geografía (2014). Estadísticas a propósito del Día Internacional de las Personas de Edad. México: INEGI. Recuperado de http://www.inegi.org.mx/saladeprensa/aproposito/2014/adultos0.pdf.

Instituto Nacional de Estadística y Geografía (2017). Estadísticas a propósito del Día Internacional de las Personas de Edad. México: INEGI. Recuperado de http://www.inegi.org.mx/saladeprensa/aproposito/2017/edad2017_Nal.pdf.

Instituto Nacional de las Personas Adultas Mayores (2018). Conoce las acciones y programas de INAPAM para nuestros adultos mayores. México: INAPAM. Recuperado de https://www.gob.mx/sedesol/articulos/conoce-las-acciones-y-programas-de-inapam-para-nuestros-adultos-mayores.

Montes de O., Z., V. (2013). La discriminación hacia la vejez en la Ciudad de México: contrastes sociopolíticos y jurídicos a nivel nacional y local. Perspectivas Sociales, 15(1), 47-80.

Organización Mundial de la Salud (2012). Estadísticas Sanitarias Mundiales. Ginebra: OMs. Recuperado de http://www.who.int/ gho/publications/world_health_statistics/2012/es/.

Organización Mundial de la Salud (2014). Prevención del suicidio. Un imperativo global. Ginebra: OMS. Recuperado de http:// www.who.int/mental_health/suicide-prevention/world_report_2014/es/.

Portal M., J. y Montano D., M. (2009). Mortalidad por suicidio en adultos mayores, provincia Pinar del Río. Revista de Ciencias Médicas de Pinar del Río, 13(1), 11-22. Recuperado de http://scielo.sld.cu/scielo.php?script=sci_arttextypi$\mathrm{d}=\mathrm{S} 1561-31942009000100002 \mathrm{ylng}=$ esytlng=es.

Ramos P., S. (2015). Una propuesta de actualización del modelo biopsicosocial para ser aplicado a la esquizofrenia. Revista de la Asociación Española de Neuropsiquiatría, 35(127), 511-526. doi: 10.4321/S0211-57352015000300005.

Ribot R., V., Alfonso R., M., Ramos A., M., y González C., A. (2012). Suicidio en el adulto mayor. Revista Habanera de Ciencias Médicas, 11(Supl. 5), 699-708.

Sánchez Z., A., y Gómez G., C. (2002). Intento de suicidio en la enfermedad de Huntington. Salud en Tabasco, 8(2), 87-90. Recuperado de http://www.redalyc.org/articulo.oa?id=48708208.

Velásquez S., J.M. (2013). Suicidio en el anciano. Revista Colombiana de Psiquiatría, 42(1), 80-84. 